\title{
Decimal-by-decimal analysis of the gravitational constant value as exemplified by torsion balance
}

\author{
Kiryan D. G., Kiryan G. V. \\ Institute of Problems of Mechanical Engineering of RAS \\ 61 Bolshoy Prospect V.O., 199178, Saint Petersburg, Russia \\ e-mail:diki.ipme@gmail.com
}

Term "gravitational constant" was for the first time introduced more than 200 years ago, and since that time attempts have been made to refine its value. As per the materials of Committee on Data for Science and Technology (CODATA), all indirect measurements of the "gravitational constant" obtained by various research groups exhibit in the SI system equality of two first decimals and spread in subsequent decimals. We have analyzed this situation by using the torsion balance mathematical model. This paper shows that this situation might be explained by solving the direct metrological problem, namely, calculation of the necessary measurement accuracies of each of the torsion balance parameters from the preset accuracy of the "gravitational constant" value. Decimal-by-decimal analysis of the torsion balance sensitivity, jointly with the CODATA data, has lead us to the assumption that all the variety of the "gravitational constant" values was obtained at experimental setups without appropriately planning the final result accuracy.

\section{Problem definition}

In his work "Traitè de mècanique" (1809), Simèon Denis Poisson introduced into the law of gravitational interaction between two material bodies factor $\mathbf{G}$ named "gravitational constant"1. The law states that the force of gravitational interaction between two homogeneous spheres (material points) is directly proportional to their gravitating ${ }^{2}$ masses and inversely proportional

\footnotetext{
${ }^{1}$ The history of the "gravitational constant" is presented in detail in the K. F. Tomilin's book "Fundamental physical constants in the historical and methodological aspects" [1, pp. 106-126]

${ }^{2}$ The concept of the material body gravitating mass is considered in papers $[2,3]$. Briefly speaking, the material body gravitating mass $\widetilde{m}$ is a function of density of its material environment. The gravitating mass functional expression looks as follows:

$$
\widetilde{m}=m\left(1-\rho_{0} / \rho\right), \quad m>0,
$$

where $\rho_{0}$ is the medium density, $\rho$ is the averaged density of the material body, $m$ is the gravitating mass of the body at $\rho_{0}=0$ (i.e., in the absence of the material medium). Hereinafter term "gravitating" is omitted for brevity, however, it is necessary to keep in mind that the medium factor always exists and needs proper attitude from researchers.
} 
to the squared distance between their centers of symmetry. Later a scaledimension factor was introduced into the law of gravitation, which was titled "gravitational constant".

Let us consider two homogeneous spheres $m^{*}$ and $m$ in masses in the Cartesian coordinate system Oxyz (Fig. 1). Positions of the spheres are

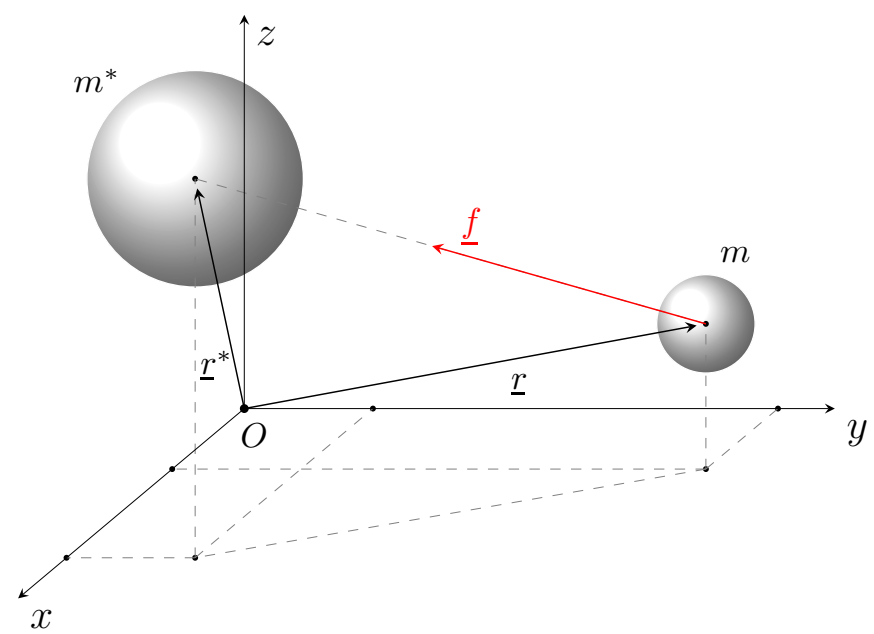

Figure 1: Gravitational force $f$ acting upon a homogeneous sphere with mass $m$ from a homogeneous sphere with mass $m^{*}$.

defined by radius-vectors $\underline{r}^{*}$ and $\underline{r}$, respectively. In this case, the law of gravitational interaction (i.e., the expression for gravitational force $\underline{f}$ acting upon mass $m$ from mass $\left.m^{*}\right)$ obtains the following form:

$$
\underline{f}=\underline{E}\left(m^{*}, \underline{r}^{*}-\underline{r}\right) m, \quad \underline{E}\left(m^{*}, \underline{r}^{*}-\underline{r}\right)=\left(\mathbf{G} \frac{m^{*}}{\left|\underline{r}^{*}-\underline{r}\right|^{2}}\right) \frac{\underline{r}^{*}-\underline{r}}{\left|\underline{r}^{*}-\underline{r}\right|} .
$$

Here $\underline{E}$ is intensity of the gravitational field generated by mass $m^{*}$. Certainly, mass $m$ is also a source of the gravitational field and attracts mass $m^{*}$ with exactly the same force as mass $m^{*}$ attracts mass $m$.

At present, international committee CODATA ${ }^{3}$ recommends the following value of the "gravitational constant" [4]:

$$
\mathbf{G}=6.67384(80) \times 10^{-11} \mathrm{~m}^{3} /\left(\mathrm{kg} \cdot \mathrm{s}^{2}\right) .
$$

At the same time, the CODATA data [4] given in Tab. 1 demonstrate that all the "gravitational constant" values coincide only in two first decimals.

\footnotetext{
${ }^{3}$ Committee on Data for Science and Technology \& The CODATA Task Group on Fundamental Constants. http://www. codata.org
} 
TABLE XXIV Summary of values of $G$ used to determine the 2010 recommended value

\begin{tabular}{llll}
\hline \hline $\begin{array}{l}\text { Item } \\
\text { number }\end{array}$ & $\begin{array}{l}\text { Value } \\
\left(10^{-11} \mathrm{~m}^{3} \mathrm{~kg}^{-1} \mathrm{~s}^{-2}\right)\end{array}$ & $\begin{array}{l}\text { Relative } \\
\text { standard } \\
\text { uncertainty } u_{\mathrm{r}}\end{array}$ & Identification \\
\hline$G 1$ & $6.67248(43)$ & $6.4 \times 10^{-5}$ & NIST-82 \\
$G 2$ & $6.6729(5)$ & $7.5 \times 10^{-5}$ & TR\&D-96 \\
$G 3$ & $6.67398(70)$ & $1.0 \times 10^{-4}$ & LANL-97 \\
$G 4$ & $6.674255(92)$ & $1.4 \times 10^{-5}$ & UWash-00 \\
$G 5$ & $6.67559(27)$ & $4.0 \times 10^{-5}$ & BIPM-01 \\
$G 6$ & $6.67422(98)$ & $1.5 \times 10^{-4}$ & UWup-02 \\
$G 7$ & $6.67387(27)$ & $4.0 \times 10^{-5}$ & MSL-03 \\
$G 8$ & $6.67228(87)$ & $1.3 \times 10^{-4}$ & HUST-05 \\
$G 9$ & $6.67425(12)$ & $1.9 \times 10^{-5}$ & UZur-06 \\
$G 10$ & $6.67349(18)$ & $2.7 \times 10^{-5}$ & HUST-09 \\
$G 11$ & $6.67234(14)$ & $2.1 \times 10^{-5}$ & JLA-10 \\
\hline \hline
\end{tabular}

Table 1: Values of the "gravitational constant" obtained by different research groups [4].

General experience in purposeful and conscious instrumental observation of planet orbits, galaxies dynamics [5], tides on land and sea, and the Earth's gravitational field strength allows us to state that gravitational interaction between material bodies depends exclusively on their masses, geometry and distance between them. In other words, the observed gravitational interaction is defined only by the character of the physical-spatial distribution of the material medium as a whole.

With the passage of time the method of refining the "gravitational constant" became a separate challenge. The more than 200-year history of improving the techniques an increasing the instrumental sensitivity has not resulted in at least the naturally expected asymptotic refining of constant $\mathbf{G}$. In the SI system, only two first decimals of the $\mathbf{G}$ values obtained by each research group are equal. As an example of a commonly accepted approach to indirect measurement of the "gravitational constant", paper "Measurement of Newton's Constant Using a Torsion Balance with Angular Acceleration Feedback" [6] may be considered.

What is the practical meaning of the difference in the third and subsequent decimals? What is the reason for this almost two-century epic of the fruitless search for the precise value of "gravitational constant" G by indirect measurements? Let us consider the current situation from the point of view of both our main goal (refining of the "gravitational constant") and the available capabilities. For this purpose, let us solve the direct metrological problem. 


\section{The torsion balance schematic model}

As a basic model, we have taken the simplest torsion balance design that allows the mass-to-mass gravitational interaction to be detected via the static torsion angle of the quartz fiber. Transient oscillation processes are neglected. We will not analyze other structural and methodological versions of indirect "gravitational constant" measurements since, as shown further, they have the same disadvantages as the classical measurement scheme based on measuring the fiber torsion angle.

Fig. 2 presents the torsion balance schematic model in the Cartesian coordinate system $O x y z$. Axis $O z$ is perpendicular to the picture plane and directed toward the reader. Weightless and rigid beam $A_{1} A_{2}$ with two equal masses $m_{A}$ at the ends is suspended at point $O$ by using a weightless and tensionless quartz fiber $L$ in length and $d$ in diameter. The beam arms are equal to each other: $\left|O A_{1}\right|=\left|O A_{2}\right|=h$. The plane of the beam $A_{1} A_{2}$ rotation about point $O$ is perpendicular to the vector of the Earth's gravitational field intensity $\underline{E}_{\text {Terra }}$.

The controllable gravitational action on the torsion balance masses is realized through two motionless masses $m_{B}$ located at points $B_{1}$ and $B_{2}$ on the circle of radius $h$. Orientation of the motionless masses $m_{B}$ is defined by angle $\beta$ between axis $O x$ and the line connecting points $B_{1}$ and $B_{2}$. As the positive direction, the counterclockwise rotation is taken. In addition, let us take into account the effect of gravitational anomaly $m_{C}$ located outside the torsion balance at the distance $h_{C}$ from point $O$ with azimuth $\varphi$ that is the angle between axis $O x$ and straight line $O C$. To prevent displacement of the point where the quartz fiber is connected to the beam with respect to motionless masses $m_{B}$, let us forbid the point $O$ motion within plane $O x y$. This will cause an appropriate response at point $O$, but it will not affect the static equilibrium of the system.

Static equilibrium of beam $A_{1} A_{2}$ is characterized by angle $\alpha$, therefore, positions of moving masses $m_{A}$ at points $A_{1}$ and $A_{2}$ may be defined by the following radius-vectors:

$$
\underline{r}_{A_{1}}=h\left(\begin{array}{c}
\cos (\pi+\alpha) \\
\sin (\pi+\alpha) \\
0
\end{array}\right), \quad \underline{r}_{A_{2}}=h\left(\begin{array}{c}
\cos (\alpha) \\
\sin (\alpha) \\
0
\end{array}\right) .
$$

Positions of the motionless masses at points $B_{1}$ and $B_{2}$ are defined in the similar way:

$$
\underline{r}_{B_{1}}=h\left(\begin{array}{c}
\cos (\pi+\beta) \\
\sin (\pi+\beta) \\
0
\end{array}\right), \quad \underline{r}_{B_{2}}=h\left(\begin{array}{c}
\cos (\beta) \\
\sin (\beta) \\
0
\end{array}\right),
$$




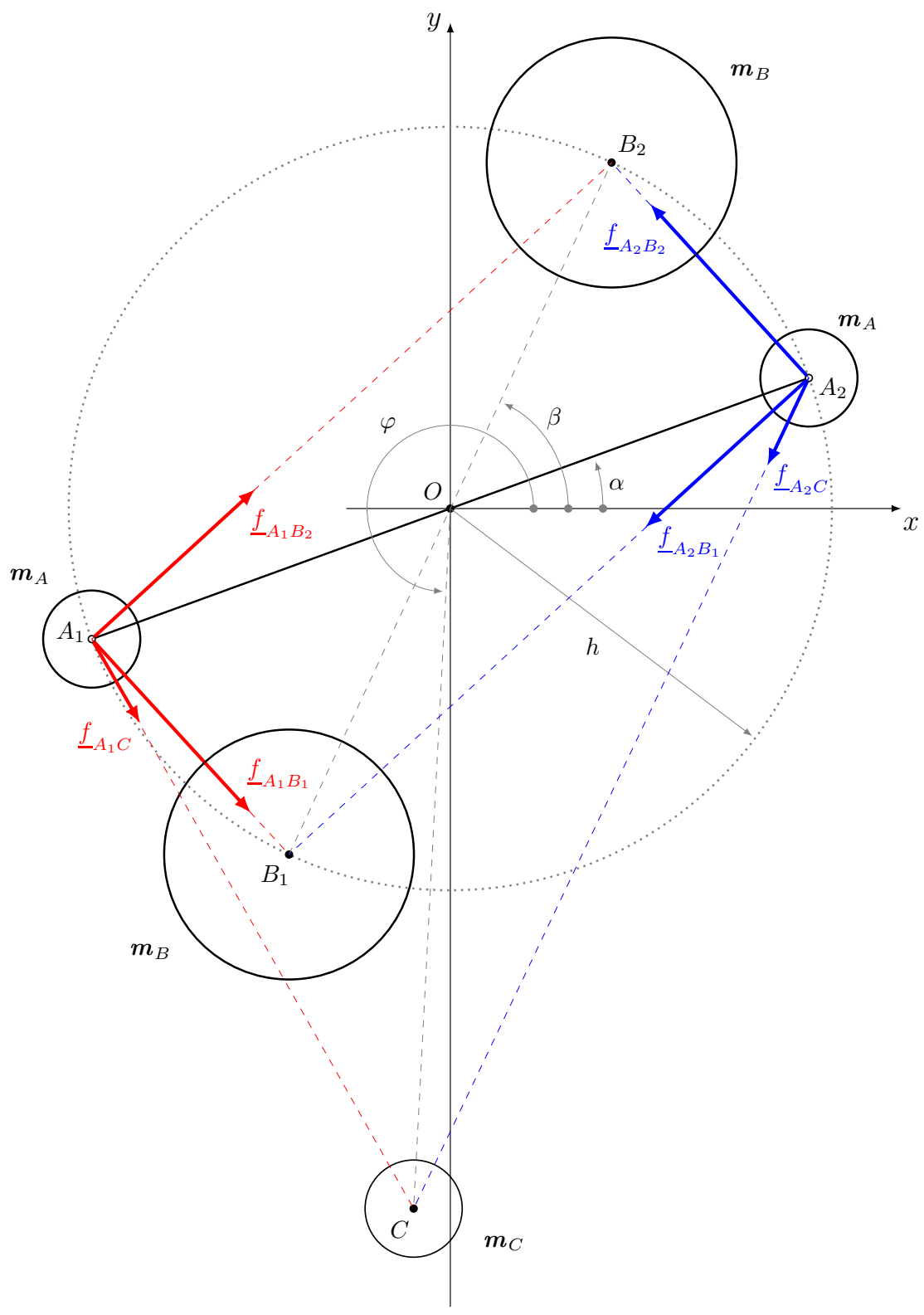

Figure 2: The torsion balance schematic model.

as well as the radius-vector of gravitational anomaly $m_{C}$ :

$$
\underline{r}=h_{C}\left(\begin{array}{c}
\cos (\varphi) \\
\sin (\varphi) \\
0
\end{array}\right)
$$

When the system is in equilibrium, this means that the sum of all the torsional moments acting on the quartz suspension fiber is equal to zero.

Kiryan D. G., Kiryan G. V. ( gconst7, v7 beta 5d.en ) - November 20, 2017 
The beam suspension fiber response to torsion manifests itself through the elastic force moment $\underline{M}_{\alpha}$ and may be expressed as follows:

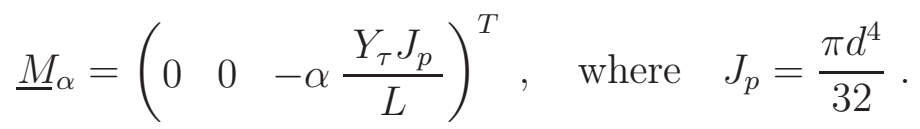

Here $\alpha$ is the suspension fiber torsion angle, $Y_{\tau}$ is the shear modulus of the fiber material [7], $J_{p}$ is the polar moment of inertia of the round fiber crosssection, and $L, d$ are the suspension fiber length and diameter, respectively.

The moments of gravitational forces twisting the suspension fiber may be subdivided into two components: the moment of gravitational action from motionless masse $\underline{M}_{\beta}$ and moment from gravitational anomaly $\underline{M}_{\varphi}$.

Taking into account axial symmetry of the masses (Fig. 2), let us consider gravitational interaction between mass $m_{A}$ with radius-vector $\underline{r}_{A_{1}}$ and two motionless masses $m_{B}$ with radius-vectors $\underline{r}_{B_{1}}$ and $\underline{r}_{B_{2}}$, and then double the moment:

$$
\underline{M}_{\beta}(\alpha)=2 \cdot \underline{r}_{A_{1}} \times\left(\underline{f}_{A_{1} B_{1}}+\underline{f}_{A_{1} B_{2}}\right),
$$

where

$$
\begin{aligned}
\underline{f}_{A_{1} B_{1}} & =\underline{E}\left(m_{B}, \underline{r}_{B_{1}}-\underline{r}_{A_{1}}\right) m_{A}, \\
\underline{f}_{A_{1} B_{2}} & =\underline{E}\left(m_{B}, \underline{r}_{B_{2}}-\underline{r}_{A_{1}}\right) m_{A} .
\end{aligned}
$$

Now let us introduce the effect of gravitational anomaly $m_{C}$ on the masses located at the ends of the $A_{1} A_{2}$ beam:

$$
\begin{aligned}
\underline{f}_{A_{1}} & =\underline{E}\left(m_{C}, \underline{r}-\underline{r}_{A_{1}}\right) m_{A}, \\
\underline{f}_{A_{2}} & =\underline{E}\left(m_{C}, \underline{r}-\underline{r}_{A_{2}}\right) m_{A} .
\end{aligned}
$$

Due to geometrical asymmetry, forces $f_{A_{1}}$ and $\underline{f}_{A_{2}}$ cause additional rotation of the beam and its displacement in the Oxy plane. Within our task, only the additional contribution of gravitational anomaly $m_{C}$ to the suspension fiber torsion angle is of interest. Therefore, displacement of point $O$, i.e., of the point where the beam is suspended, is forbidden $\left(\left|\underline{r}_{O}\right|=0\right)$.

Thus the torsional moment induced by gravitational anomaly $m_{C}$ with radius-vector $\underline{r}_{C}$ takes the following form:

$$
\underline{M}_{\varphi}(\alpha)=\underline{r}_{A_{1}} \times \underline{f}_{A_{1} C}+\underline{r}_{A_{2}} \times \underline{f}_{A_{2} C} .
$$

The torsion balance is in the static equilibrium, which means that the sum of gravitational moments $\underline{M}_{\beta}$ eq. (7), $\underline{M}_{\varphi}$ eq. (10) and elastic force moment $\underline{M}_{\alpha}$ eq. (6) is zero:

$$
\underline{M}_{\alpha}(\alpha)+\underline{M}_{\beta}(\alpha)+\underline{M}_{\varphi}(\alpha)=0 \text {. }
$$


This transcendental equation gives us the static torsion angle $\alpha$ and thus establishes the interrelation between the beam rotation angle $\alpha$ and torsion balance parameters. Angle $\alpha$ is bounded from above, the limiting value being dependent on the schematic model geometry (Fig. 2). The maximum permissible angle of the beam $A_{1} A_{2}$ rotation may be defined as

$$
\alpha_{\max }=\beta-\arccos \left(1-\frac{1}{2}\left(\frac{R_{A}+R_{B}}{h}\right)^{2}\right),
$$

where $\alpha_{\max }$ is the maximum permissible rotation angle of the beam, $R_{A}, R_{B}$ are the radii of the moving and motionless spheres, respectively.

To continue analyzing the accuracy of the preset torsion balance parameters, let us deduce "gravitational constant" G from (11):

$$
\mathbf{G}=\alpha \frac{Y_{\tau}}{L} \frac{\pi d^{4}}{32} \frac{2 h}{m_{A} m_{B}}\left(\frac{\cos \xi}{\sin ^{2} \xi}-\frac{\sin \xi}{\cos ^{2} \xi}\right)^{-1}, \quad \xi=\frac{\beta-\alpha}{2} .
$$

This relation enables determination of the necessary accuracy of each of the torsion balance key parameters which ensures the required accuracy of the "gravitational constant" value. Since we are interested in the accuracy characteristics of just the torsion balance with calibrated masses $m_{A}$ and $m_{B}$, we have neglected the anomaly $m_{C}$ gravitational effect on masses $m_{A}$ in deducing relation (13). The effect of gravitational anomaly on the suspension fiber torsion angle will be considered later, after solving the main problem.

\section{Calculation of the necessary accuracy of the system parameters}

Let us set the required calculation accuracy of the "gravitational constant" value and calculate the necessary measurement error in the beam rotation angle and also in key design and physical/mechanical parameters of the torsion balance.

As the required calculation accuracy of the "gravitational constant" in the SI system, we regard the number of significant decimals. Thus we assign increment $\Delta \mathbf{G}$ to each decimal in the "gravitational constant" value:

$$
\Delta \mathbf{G}=[0.1 ; 0.01 ; 0.001 ; 0.0001 ; \ldots] \times 10^{-11} \mathrm{~m}^{3} /\left(\mathrm{kg} \cdot \mathrm{s}^{2}\right) .
$$

Being under the action of the gravitational forces induced by masses $m_{B}$, the initially weightless beam with masses $m_{A}$ at the ends is in static equilibrium at the angle $\alpha$ to the $O x$ axis. Angle $\alpha$ can be found from equation (13) by using the unperturbed "gravitational constant". 
After setting the "gravitational constant" increment $\Delta \mathbf{G}$ by selecting it from sequence (14), we can find a corresponding correction to the chosen torsion balance parameter which will completely compensate the effect of the preset $\mathbf{G}$ perturbation, namely, preserve the initial static equilibrium characterized by angle $\alpha$. This compensative correction will be just the maximum permissible absolute error in the chosen parameter of the system.

First let us estimate the necessary measurement accuracy of torsion angle $\alpha$. Let us vary "gravitational constant" $\mathbf{G}$ in (6) by $\Delta \mathbf{G}$ and find a new torsion angle different from the initial one. Designate this difference as $\Delta \alpha$. This means that to calculate the "gravitational constant" with the preset accuracy $\Delta \mathbf{G}$ it is necessary to have instruments able to measure the suspension fiber torsion angle ${ }^{4}$ with absolute accuracy $\Delta \alpha$.

After that, maintaining the static equilibrium characterized by invariable torsion angle $\alpha$ eq. (13), let us find the interrelation between the preset "gravitational constant" increment $\Delta \mathbf{G}$ and corresponding compensative corrections to the basic torsion balance parameters, namely, $m_{B}, L, d$ and $Y_{\tau}$. Tab. 2 shows the torsion balance parameters used in the schematic model (Fig. 2). Calculations of the necessary measurement accuracy of the torsion

Table 2: Basic parameters of the torsion balance.

\begin{tabular}{ll}
\hline$L=0.7 \mathrm{~m}$ & - length of the beam suspension fiber; \\
$d=50 \mu \mathrm{m}$ & - suspension fiber diameter; \\
$h=100 \mathrm{~mm}$ & - beam arm length; \\
$Y_{\tau}=31.1966 \mathrm{GPa}$ & - shear modulus of the suspension fiber (material: $\mathrm{SiO}_{2}$ ); \\
$R_{A}=10 \mathrm{~mm}$ & - radius of the spheres (moving masses) at the beam ends; \\
$m_{A}=0.0821 \mathrm{~kg}$ & - moving sphere mass (material: W); \\
$R_{B}=70 \mathrm{~mm}$ & - radius of the spheres (motionless masses); \\
$m_{B}=28.1604 \mathrm{~kg}$ & - motionless sphere mass (material: W); \\
$\beta=57^{\circ}$ & - angle determining location of motionless masses $m_{B} ;$ \\
\hline
\end{tabular}

\footnotetext{
${ }^{4}$ In case the interacting material bodies $m_{A}$ and $m_{B}$ are homogeneous spheres, the beam suspension torsion angle is essentially a strict angular equivalent of the distance between geometric centers of the spheres and hence it is quite reasonable to use relation (1) to calculate the "gravitational constant".

When the pair of masses consists of bodies with the shape different from spherical (e.g., cylinders), there arises a problem with applying the law of universal gravitation (1) since in this case the gravitational interaction force does not correlate with the distance between the cylinder's centers of mass. In this case correction factors should be used which arise from an approximate solution of the problem of gravitational interaction between cylindrical material bodies, which, in its turn, contributes additional uncertainty in the "gravitational constant" calculations.
} 
balance parameters are listed in Tab. 3. The results obtained are shown in

Table 3: Maximum permissible absolute measurement errors of the torsion balance parameters for various values of the "gravitational constant" absolute accuracy.

\begin{tabular}{c|rrrrr}
\hline$\Delta \mathbf{G} \times 10^{-11}$ & $\Delta \alpha, \operatorname{arcsec}$ & $\Delta m_{B}, g$ & $\Delta L, m m$ & $\Delta d, \mu m$ & $\Delta Y_{\tau}, G P a$ \\
\hline 0.1 & 662.654 & 415.696 & 10.333 & 0.18624 & 0.46741 \\
0.01 & 65.541 & 42.129 & 1.047 & 0.01872 & 0.04674 \\
0.001 & 6.547 & 4.219 & 0.105 & 0.00187 & 0.00467 \\
0.0001 & 0.655 & 0.422 & 0.010 & 0.00019 & 0.00047 \\
0.00001 & 0.065 & 0.042 & 0.001 & 0.00002 & 0.00005 \\
\hline
\end{tabular}

Figs. 3,4 that clearly demonstrate a significant increase in the required measurement accuracy of the torsion balance basic parameters with increasing number of decimals in the "gravitational constant" value. The maximal

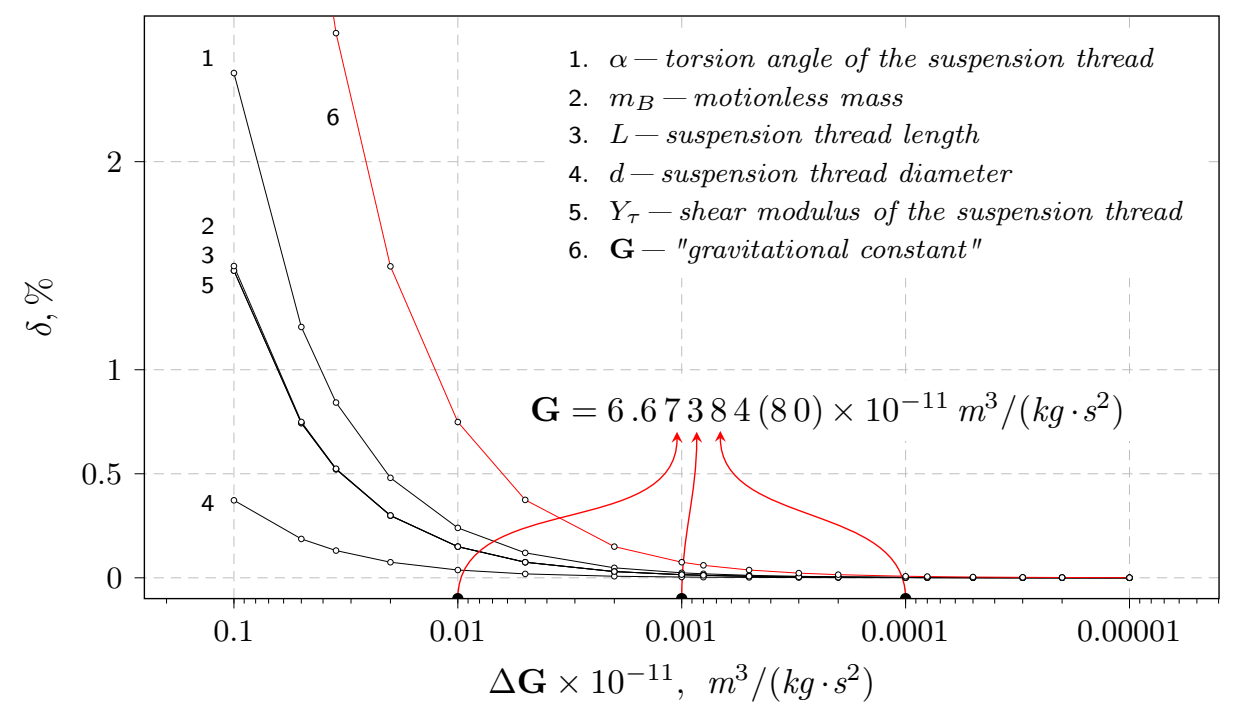

Figure 3: Relative accuracy of the schematic model parameters versus the number of decimals in the "gravitational constant" value.

error of the "gravitational constant" indirect measurement is graphically represented in Figs. 3,4. The error was calculated via the following formula:

$$
\delta \mathbf{G}=\frac{1}{\mathbf{G}} \sum_{i=1}^{5}\left|\frac{\partial \mathbf{G}}{\partial q_{i}}\right| \Delta q_{i}, \quad \text { where } q_{i}=\left\{\alpha, m_{B}, L, d, Y_{\tau}\right\} .
$$

Based on the data of Tab. 3, Figs. 3,4 and also on the fact that errors are accumulative as relation (15) shows, it is possible to define an empiric rule 


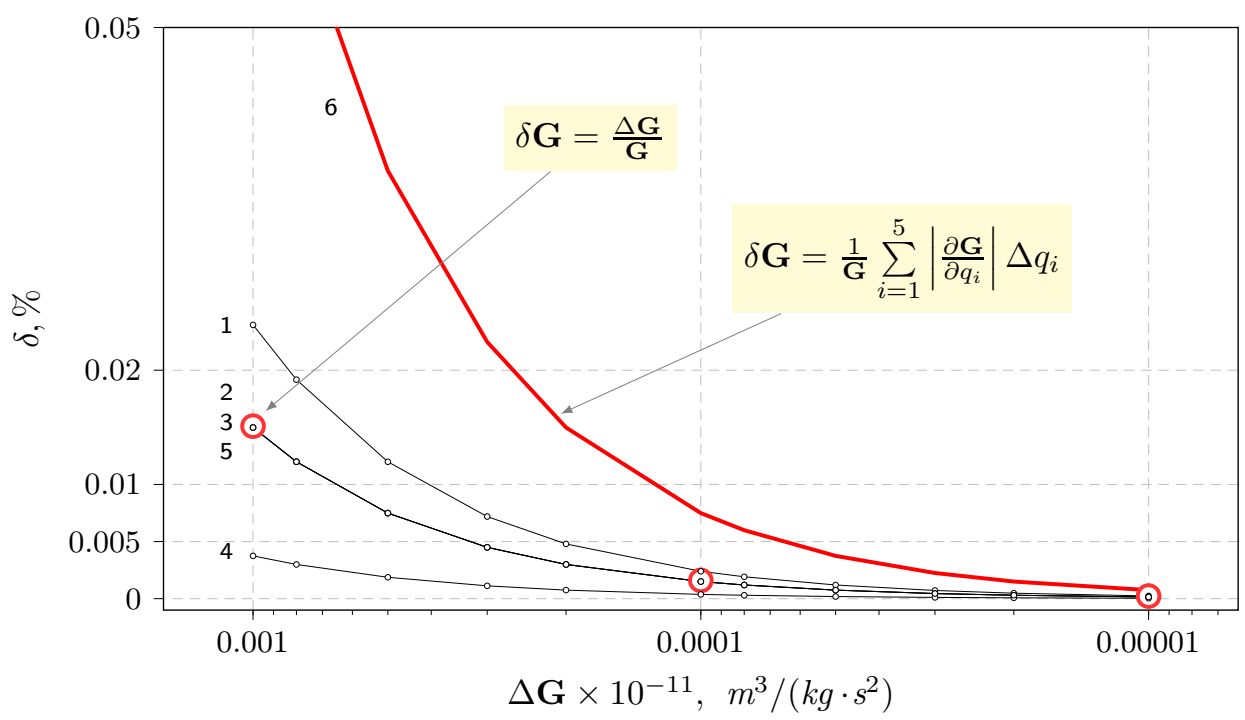

Figure 4: A Figure 3 fragment presented in a larger scale.

stating that when the "gravitational constant" is found with the accuracy of, e.g., $\Delta \mathbf{G}=0.001 \times 10^{-11} \mathrm{~m}^{3} /\left(\mathrm{kg} \cdot \mathrm{s}^{2}\right)$, the maximum permissible absolute measurement error of each torsion balance parameter should be calculated based on the $\Delta \mathbf{G}$ value lower by an order of magnitude.

It is possible to reject direct measurement of parameters $L, d, Y_{\tau}$ and replace them with only one parameter, i.e., torsional stiffness factor. For this purpose it is necessary to conduct an additional experiment in which masses $m_{B}$ are removed in-situ without violating the experimental setup integrity. In the course of this experiment the actual suspension fiber torsional stiffness was determined via the fixed period of the beam torsion motion. However, in this case the measurement accuracy of such parameters as mass, torsion angle, linear dimensions and time (the torsion oscillation period) still remains problematic.

The effect of gravitational anomaly $m_{C}$ on the torsion angle $\alpha$. Now let us estimate the effect of gravitational anomaly $m_{C}$ on the experimentally measured torsion angle of the beam $A_{1} A_{2}$ suspension fiber (Fig. 2). Assume that when anomaly $m_{C}$ was absent, the torsion balance beam was turned by angle $\alpha$. Using the torsional moment balance equation (11), calculate the new static torsion angle $\alpha_{C}$ allowing for the presence of gravitational anomaly $m_{C}$ at point $C$ defined by radius-vector $\underline{r}_{C}$. The asymmetric location of mass $m_{C}$ gives rise to an additional torsional moment and response at point $O$. Here we consider only the torsional moment since within our task beam $A_{1} A_{2}$ can rotate only in plane $O x y$ about point $O$. Tab. 4 lists the 
Table 4: The effect of gravitational anomaly $\left(m_{C}=100 \mathrm{~kg}\right.$, azimuth $\left.\varphi=90^{\circ}\right)$ on the suspension fiber torsion angle.

\begin{tabular}{c|cc}
\hline$\left|\underline{r}_{C}\right|, m$ & $\left(\alpha_{C}-\alpha\right), \operatorname{arcsec}$ & $\delta \alpha, \%$ \\
\hline 2 & 6.4526091 & 0.0236213 \\
3 & 1.9179015 & 0.0070209 \\
5 & 0.4149482 & 0.0015190 \\
10 & 0.0519051 & 0.0001900 \\
30 & 0.0019228 & 0.00000070 \\
Moon & 0.0008894 & 0.0000033 \\
50 & 0.0004153 & 0.0000015 \\
\hline
\end{tabular}

calculations reflecting the effect of the gravitational anomaly on the torsion angle of the beam suspension fiber. Analysis of the Tab. 4 data unambiguously shows that it is necessary to ensure local axial symmetry of the gravitational field within a radius of minimum 5 meters from the suspension fiber of the torsion balance beam. As for celestial bodies, in the framework of our schematic model such gravitational anomalies as e.g. Moon (in perigee) produce extremely minor (instrumentally non-measureable) effect on the suspension fiber torsion angle. Thus celestial bodies may be excluded from consideration.

\section{Conclusions}

Solving of the direct metrological problem by using the torsion balance mathematical model indeed gives an answer on the question why there has been no progress in refining the "gravitational constant" value during so many years and why it can hardly be expected in the foreseeable future. The decimalby-decimal analysis has shown that the current problems with the accuracy of determining the "gravitational constant" are caused by a quite real and ordinary fact, i.e., by limited metrological capacity of experimental setups in combination with methodological stereotypes.

To reach the preset accuracy of the "gravitational constant", the experimentalist should artificially increase it by an order of magnitude and then find the maximum permissible measurement errors of the system key parameters corresponding to this increased accuracy. Just these measurement errors will enable calculation of the "gravitational constant" with that preset accuracy provided the laboratory metrological capability is sufficiently high.

In addition, note that the gravitational effect of celestial bodies may be ignored due its insignificance but only under the condition of the gravita- 
tional field axial symmetry with respect to the suspension fiber of the torsion balance beam.

To summarize the above, we can state that at present the "gravitational constant" has been calculated accurately to the second decimal, and the spread in subsequent decimals (see Tab. 1) is caused by specific features of particular experimental setups and metrological capabilities of laboratories, experimental techniques, external factors, etc. Thus the process of refining the "gravitational constant" has turned into senseless waste of time and resources though the search for experimentum crucis $^{5}$ for indirect measurement of the "gravitational constant" seems to be important and useful from the metrological and engineering point of view.

\section{References}

[1] Томилин К. А. Фундаментальные физические постоянные в историческом и методологическом аспектах. - ФИЗМАТЛИТ, 2006. - 368 с.

[2] Kiryan D. G., Kiryan G. V. On the Gravitational Mass // International Journal Of Applied And Fundamental Research. - 2013. - Vol. 1. URL: http://www. science-sd. com/452-24059.

[3] Kiryan D. G., Kiryan G. V. Gravitational interaction in the medium of non-zero density // e-prints viXra:1406.0128v2. - 2014. — Jul. P. 24. - URL: http://vixra.org/abs/1406.0128.

[4] Mohr P. J., Taylor B. N., Newell D. B. CODATA recommended values of the fundamental physical constants: 2010 // Reviews of Modern Physics. 2012. - Nov. - Vol. 84, no. 4. - P. 1527-1605.

[5] Kiryan D. G., Kiryan G. V. The Evolution of the System of Gravitating Bodies // e-prints viXra:1607.0390v4. - 2016. - P. 19. — URL: http: //vixra.org/abs/1607.0390.

[6] Gundlach J. H., Merkowitz S. M. Measurement of Newton's Constant Using a Torsion Balance with Angular Acceleration Feedback. — 2000. 11. - Vol. 85. - P. 2869-72.

[7] Strong J. Physical experiment technology. — Leningrad News-paper, Magazine and Book Publishing House, 1948.

\footnotetext{
${ }^{5}$ Crucial experiment - an experiment held to decide with certainty between two rival hypotheses about some matter. (http://philosophy.enacademic.com/588/crucial_ experiment)
} 